\title{
The Microblogging Evolution Based on User's Popularity
}

\author{
Tao Shaohua*
}

School of Information Engineering, Xuchang University, Xuchang, Henan, 461000, P.R. China

\begin{abstract}
The number of fans indicate the influence of user in microblogging network. Therefore, it is particularly important to study the number changes of user's fans. The variation of fans number of users was affected by the popularity and activity, based on this two factors the evolution mechanism was proposed. The followings degree distribution, average short path, betweeness and follow ration were simulated. The simulation show that weibo has both properties of small world network and scale-free network. The simulation results show that the microblogging network has the characteristics of small-world and scale-free, its bigger clustering coefficient and shorter average path length. The simulation verified the correctness of the theory.
\end{abstract}

Keywords: Fans, microblogging network, microblogging, user.

\section{INTRODUCTION}

Micro-bloging, referred to as micro-blog, also known as blog. It is an application platform based Web 2.0 that allows users to update short text (usually less than 140 words), and publicly available information to dissemination and sharing information. This form allows the user selecting a particular group to facilitate reading. Microblogging is a online social networking platform through concern mechanism sharing brief and real-time information [1-4]. The information spread mode of microblogging is not same as the traditional media that release information basis a linear spread to public, and it also unlike traditional network media that through network spread information [5-9]. It is a new information waterfall type of spread the way. This mode of transmission of the "chain reaction" with viral propagation characteristics, and this feature is based on the trust. Concern of users of weibo is no longer looking, but active interest, this way narrow the distance between people and enhance communication with other people, and enable ordinary users communicate with celebrity becoming very easy [9-12]. In the study of relevant factors about user characteristics in weibo model, the number of fans indicates the users influence degree, so it is very important to study the changes of fans number. This model based on BA probability studying two key factors that influence the fans changes of users: activity and popularity. In simple various network factors, the microblogging evolution model was proposed. The simulation results show that the user's following degree distribution follows a power law distribution truncated. Thus, the microblogging model can be fully manifested variation law of fans and users and related factors.

*Address correspondence to this author at the School of Information Engineering, Xuchang University, Henan, 461000, P.R. China;

Tel: 15038981083; E-mail: shtao_2008@126.com

\section{SINA WEIBO AND TWITTER}

\subsection{Based on Design Process and Production Process of Product Components Decomposition}

Sina Weibo developed quickly, but it has great differences with Twitter at large. The following from three aspect of update frequency, content type of users and concern to analyze difference of Twitter and Sina micro-blogging [13].

1. Update frequency: from the user's point of view update frequency, network media users more than traditional media users, while other users information update relatively few. In china user's of Sina microblogging activity far less than Twitter's users, shown as Fig. (1).

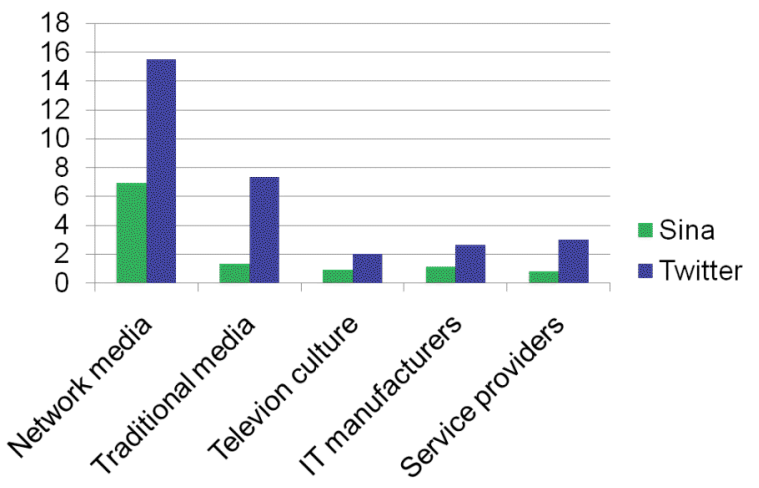

Fig. (1). Update frequency of Sina and Twitter.

2. User's content type: the network media has the highest proportion among all of online hop links web sites [13]. Among originality content, comment and reply, the IT (software) has highest proportion. The online hop links proportion reached $80 \%$ of media network, the interaction was not as good IT (software) manufacturers, shown as Fig. (2).

3. Concerned comparison: the media-user ha s the most attention, followed by the Television Culture Company, others small, shown as Fig. (3). 


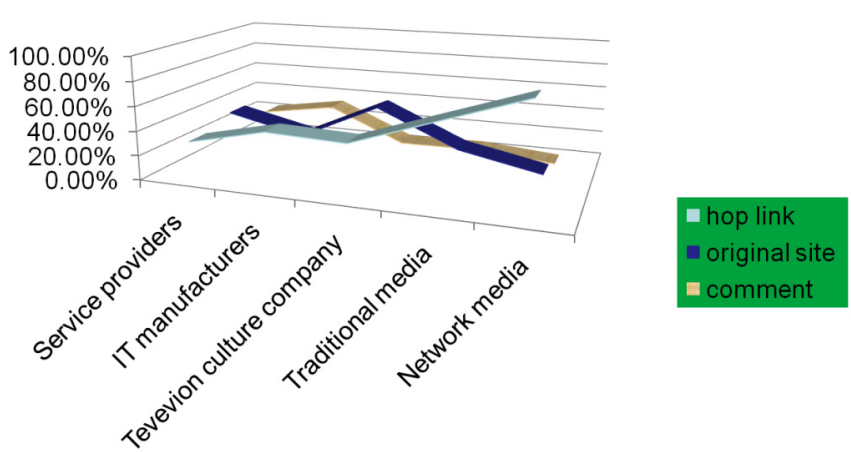

Fig. (2). User's content of microblogging.

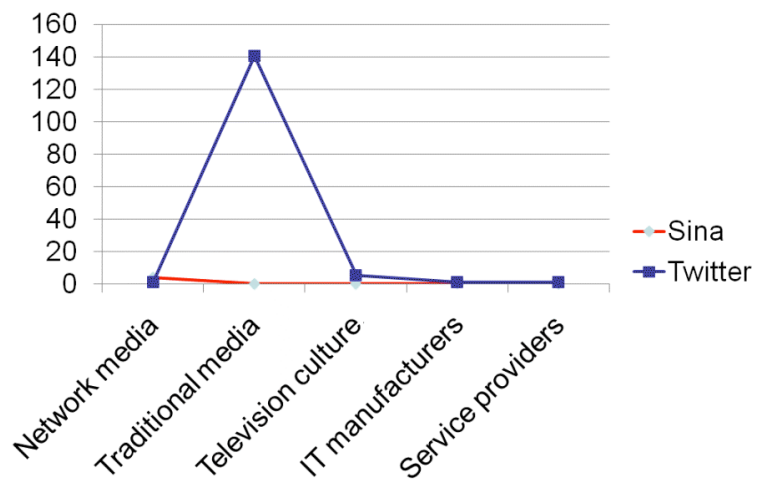

Fig. (3). Concern comparision of Sina and Twitter.

From the figure, the number of fans of Sina users is far less than Twitter; while in the user agency types, the number of network media of Sina is greater than Twitter.

The Sina Weibo contrast with Twitter, the former has the more network media attention, the latter mainly with traditional media. Overall, in institutional users, the media-users more active, while activity in the enterprise Weibo has yet to be improved.

Individual users are divided into celebrities and common users. Celebrity users can be divided into sports stars, movie stars, and so on [13]. For the organization can be divided into government, the media and so on. In this paper, the user are classified based on the Sina Weibo user's classification standards. The user represented by the following categories tree, shown as Fig. (4).

In microblogging network, the in-degree is the number of fans, and the out-degree is the number of friends. Users through the "concern" to build relationships with "being concerned". Users can concern the others without the other user's allow and can receive dynamic updates information of other users. In study related factors of Weibo model user's characteristics behavioral, the number of fans of users indicates the user's influence scope and extent, so study the changes of fans number is very important.

\subsection{Degree Distribution of the Movie Star}

Movie stars belong to the special individual users. From Sian Weibo statistics data [13], input the number of fans and use the matlab simulate the data, shown as Fig. (5). In movie star users, the probability of the same fans is very small, only $1 \%$. The in-degree distribution is equidistant steady decline curve. As can be seen from the simulation, the change of the user's number of fans are truncated power-law distribution.

\subsection{In-Degree Distribution of Organization Users}

Media has a profound impact on people's social life, this paper selected media certified in Sina microblogging as samples to study the distribution of the number of fans of Organizational users [13]. Media category: TV channels, TV programs, online media, newspapers, magazines and radio. The probability of same number of fans in each subclass appear very small, basically between $2 \%-10 \%$, same as movie star, the in-degree distribution also is power-law distribution. According to the relevant data collection, the result showed that the distribution of six Organization are truncated power-law distribution, shown as Fig. (6).

\section{MODEL PROPOSED}

\subsection{Microblogging Topology}

Research the fans changes of users in microblogging, the main study was the size of following and friends, therefore, with directed graph showing the relationship between users.

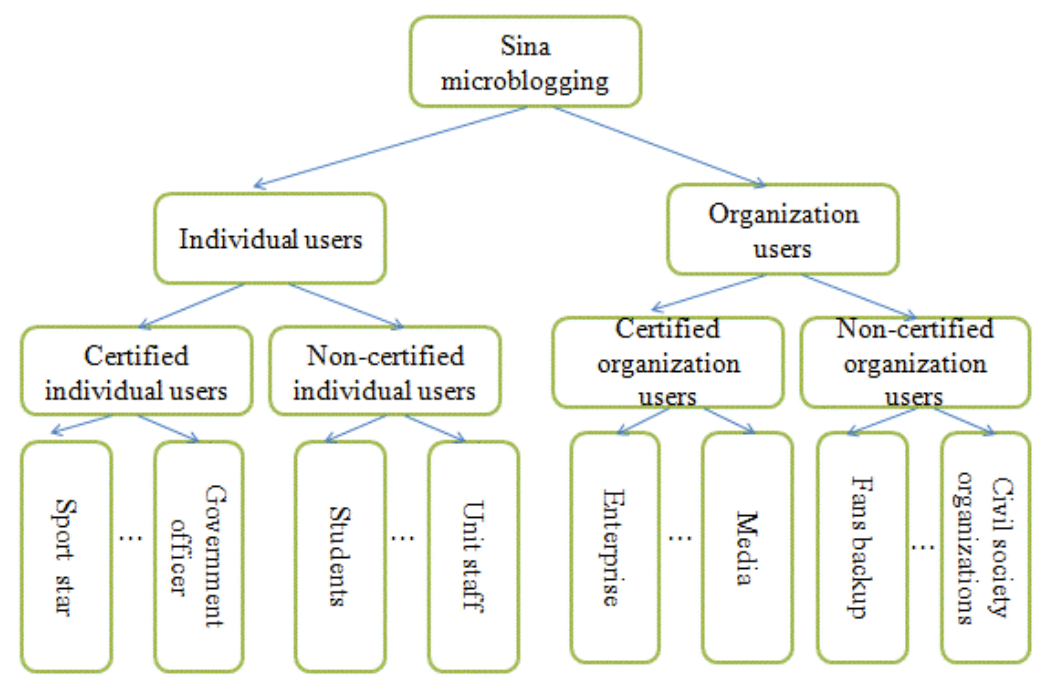

Fig. (4). User categories of Sina. 


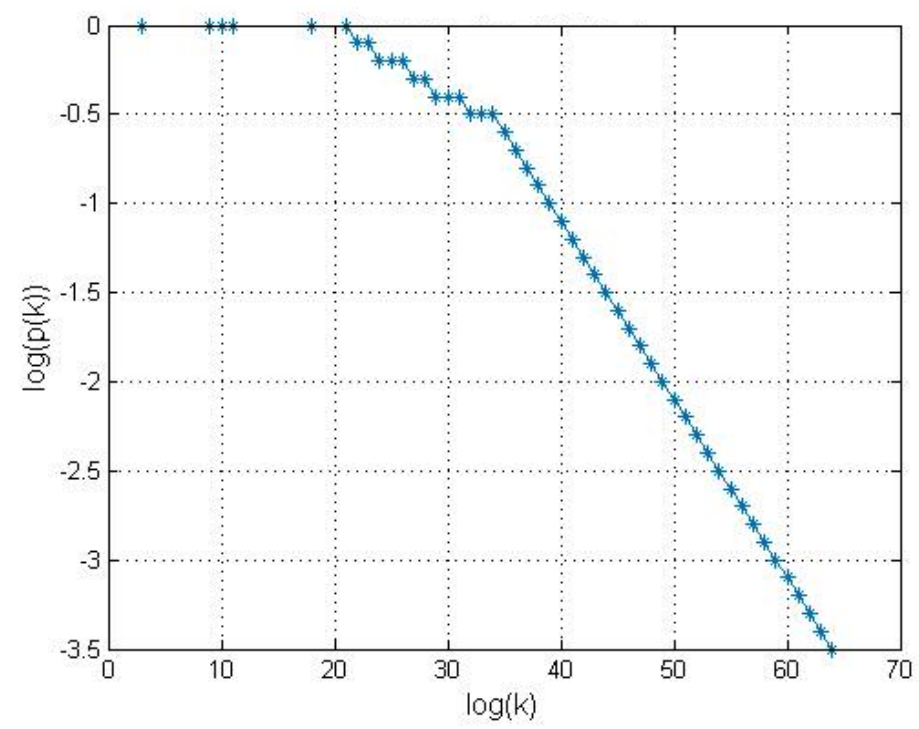

Fig. (5). Movie star's degree distribution.

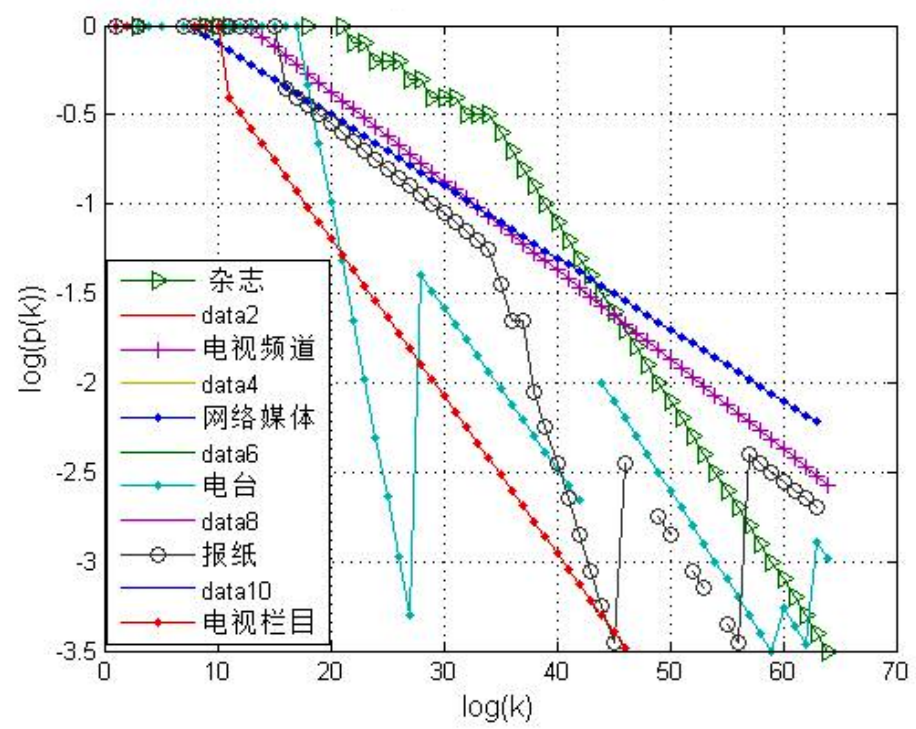

Fig. (6). Organization users degree distribution.

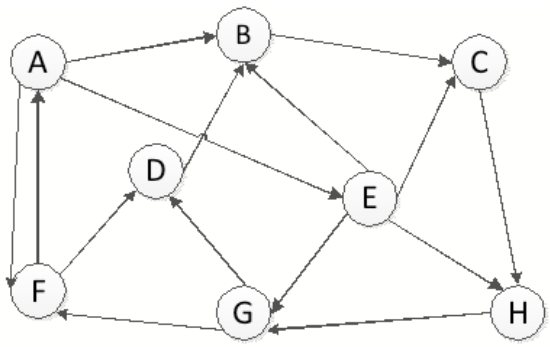

Fig. (7). Microblogging topology.

In microblogging network, each user has many fans as well as a fans of other users. it is the relationship $n-n$ that many relationship among users. The edges between users present as fan and follower in microblogging, shown as Fig. (1). Between user $B$ and user $C$ has an edge pointed from $B$ to $C$, indicating the relationship: user $B$ is a fan of user $\mathrm{C}, \mathrm{C}$ is $\mathrm{B}$ followers. Another is the relationship between user $\mathrm{A}$ and user $\mathrm{B}$ having bidirectional edges, which is "mutual reciprocity ", shown as Fig. (7).

\subsection{Influence Factor}

Influence factor which is the user's popularity. Popularity is the user update his/her weibo which attraction and influence other user's degree. A user's popularity is mainly reflected in two aspects: the number of forwarded and the number of comments.

There is a high correlation between forward and comments, comments increasing along with the forwarding increasing. The number of comments reflected the degree of response to microblogging in the diffusion process that is triggered by influence. As a reader, which is an attitude and evaluation to user's microblogging. While the number of forwarding reflected the extend of weibo diffusion. Although the number of fans is the foundation of comments and forwarding of weibo, the content of weibo is also important, such as Correla3, it has a large of fans while its comments and forwarding very few. Therefore, the most popular factor reflect the degree of the user's concern is the number of 
weibo forwarded, which can be said, the higher the popularity of the user and the greater the influence of the user.

\subsection{Microblogging Topology}

On the basis of the preferential attachment of BA network and user's different influence, the microblogging network evolving as following:

Step 1: Assuming the initial time $t_{0}=0$, the $n_{0}$ users in the network, each user's has different influence on the number of different fans.

Step 2: (Network growth): in $t$ steps, repeating add new nodes $n$ (new users) and new links $m$ (new fans). With time growing, users in network increases has two parts: one parts is microblogging network is accepted by the public and more people likes it, registered users on it increased; The other part is the new users becoming old users and attracting other users following themselves with time accumulation. These changes in the number of fans of users affected by themselves popularity.
Step 3: (Popularity link): The basis of the newly added $m$ links according to preferential attachment $\Pi_{k}$ to select. Concerned about the relationship, whether it is concerned about other people becoming fans of others, or be concerned to increase their number of fans, new and old users are to follow preferential attachment.

Step 4 (model update): In single microblogging groups, according to preferential attachment to renew the old nodes and the number of fans of each user.

\section{SIMULATION ANALYSIS}

In evolution simulation, the initial nodes of the network is $n=10$ and the total number of nodes $N=5000$. Each time added new users, the links of old users and new users affected by fans and popularity of the old users.

\subsection{Indegree Distribution}

The degree distribution show as Fig. (8), the data scattered near a straight line, while most nodes have a small

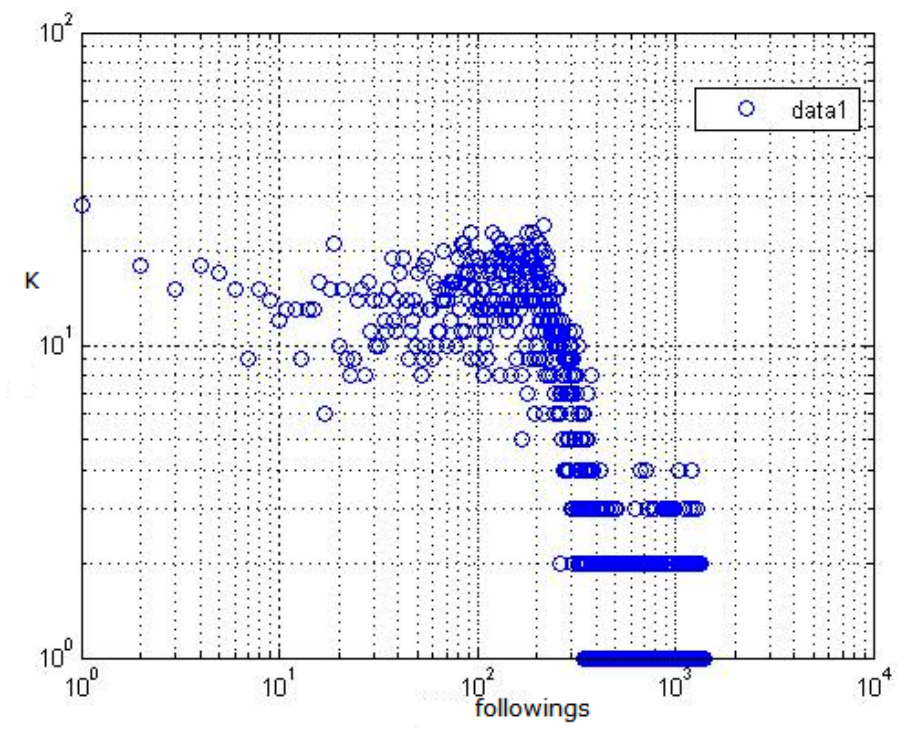

Fig. (8). Indegree distribution simulation.

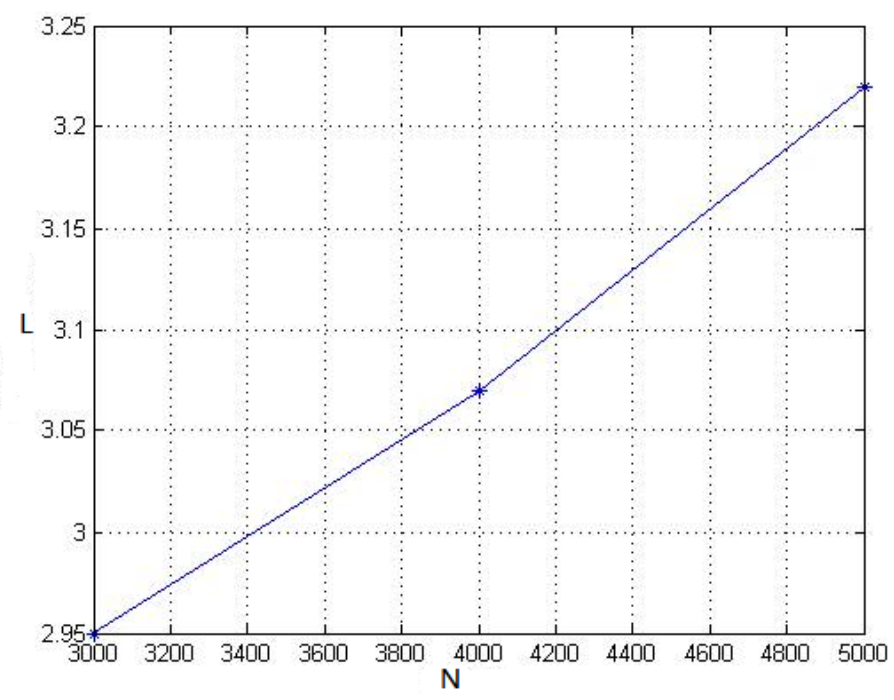

Fig. (9). Average path length. 


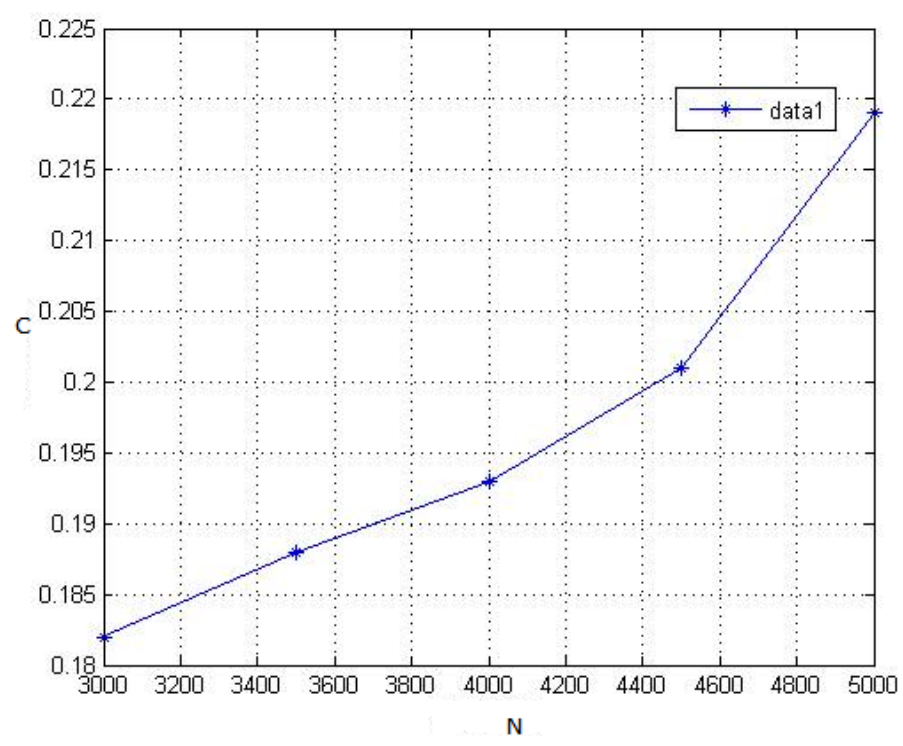

Fig. (10). Clustering of network.

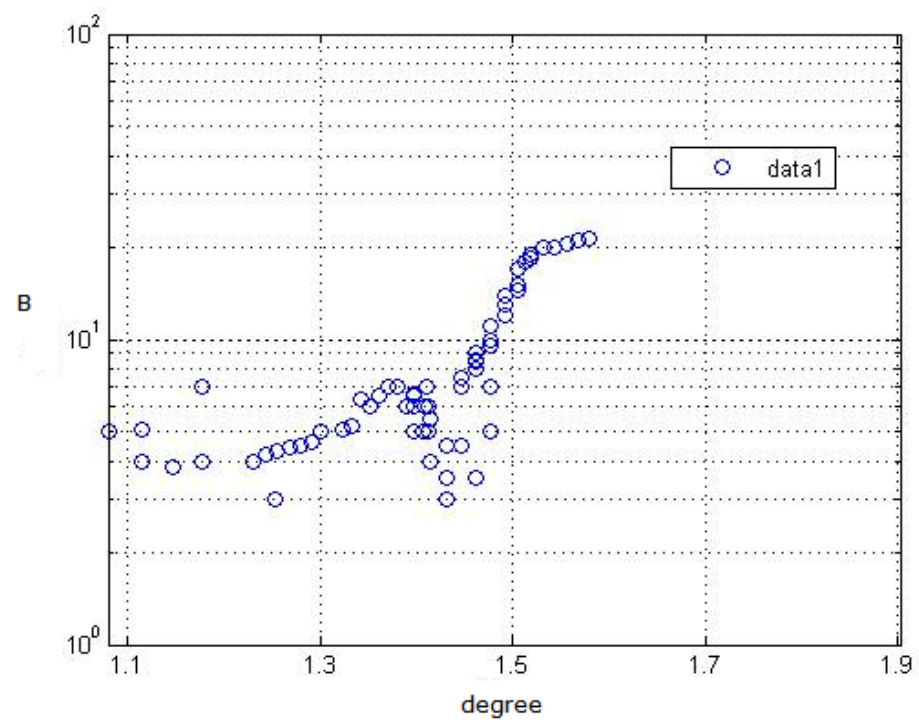

Fig. (11). Betweenness of network.

number of connections, but a few super-node has majority connections. This structural heterogeneity is precisely the same as structure of ordinary users and stars in microblogging network. The degree distribution of network nodes showed a power-law distribution, so the microblogging network has the characteristics of scale-free.

\subsection{Average Path Length}

As the number of nodes adds, the network size increasing and the average path length increase with the logarithmic growth, shown as Fig. (9). The average path length of network is about 3 , by definition, any two users in network need an average of three people can be meet. So microblogging network has a shorter average path length determining the Weibo users with small world effect, thus, the information can spread rapidly among them.

\subsection{Clustering}

Clustering coefficient $C(i)$ is to describe the probability of $i-t h$ node connected to the one pair of nodes. That is to say the link probability between two friends of one node. In this network, the clustering coefficient is about 0.2 , when the larger the network, the clustering coefficient increases and the more dense relationships in network, shown as Fig. (10).

\subsection{Betweenness}

Usually the betweeness of nodes growth with the ndoe's degree increasing, which is the nodes degree larger and the betweeness of its also larger, shown as Fig. (11). The nodes with bigger betweenness at the center of the network, and the number of shortest paths through these nodes will be more; rather small degree of a node has and betweenness also small, these nodes at the edge of the whole network. The nodes with bigger betweenness are of a core position and play an important role in network, carrying the rise and fall to the whole network. Ordinary nodes consolidate a stable development of the whole network, it can't be ignored to information quickly spread and can acquire the first message and spread it. 


\section{CONCLUSION}

In microblogging evolutionary mechanism, different number of fans to each user have significant meaning. The causes affect the number of fans changes worthy of our discussion. Therefore, this paper studies two factors affect the fans changes of each user: activity and popularity. While popularity is the main factor affecting the fans number changes of users. We compared comments and forwarding to user's micro-blog, we got that micro-blog are forwarded more times and the user have greater influence, which is micro-blog are forwarded higher times and the user's popularity is also high. Therefore, we propose a micro-blog evolving mechanism with influence factor. According to this evolutionary mechanism, we simulated indegree distribution, average path length, clustering and betweenness. Simulation verify the validity of the model.

\section{CONFLICT OF INTEREST}

The author confirms that this article content has no conflict of interest.

\section{ACKNOWLEDGEMENTS}

This work was supported by the Foundation Name under Grant No. (122102210544 and 12A510022 and 132300410349) and natural foundation fund (No. U1304403).

\section{REFERENCES}

Kumar, J. Novak, and A. Tomkins, "Structure and evolution of online social networks," In: Proceedings of the $12^{\text {th }}$ ACM SIGKDD International Conference on Knowledge Discovery and Data Mining, ACM, New York, pp. 611-617, 2006.

[2] D. Kempe, J. Kleinberg, and E. Tardos, "Maximzing the spread of influence through a social network," In: Proceedings of the $9^{\text {th }}$
ACM SIGKDD International Conference on Knowledge Discovery and Data Mining, ACM, New York, 2003, pp.137-146.

[3] R. Crane, and D. Sornette, "Robust dynamic classes revealed by measuring the response function of a social system," In: Proceedings of the National Academy of Sciences, vol. 105, pp.1564915653,2008

[4] H. Kwak, C. Lee, H. Park, and S. Moon, "What is twitter, a social network or a news media?," In: International World Wide Web Conference Committee (IW3C2), ACM, North Carolina, 2010, pp. 256-266.

[5] M. Cha, A. Mislove, and K. P. Gummadi, "A measurement-driven analysis of information propagation in the Flickr social network," In: Proceedings of the $18^{\text {th }}$ International Conference on World Wide Web, ACM, Madrid, 2009, pp. 120-130.

[6] D. Zhao, and M. B. Rosson, "How and why people twitter: the role that micro-blogging plays in informal communication at work," In: Proceedings of the ACM, Florida, pp. 243-252, 2009.

[7] E. Sun, I. Rosenn, C. Marlow, and T. Lento, "Gesundheit! modeling contagionthrough facebook news feed," In: Proceedings of International AAAI Conference on Weblogs and Social Media, San Jose, California, 2009, pp. 61-70.

[8] D. Strang, and S. Soule, "Diffusion in organizations and social movements: From hybrid corn to poison pills," Annual Review of Sociology, vol. 24, pp.265-290, 1998.

[9] L. Gao, C. Song, Z. Gao, A. Barabasi, J. P. Bagrow, and D. Wang, "Quantifying information flow during emergencies," Science Reports, vol.6, pp. 3997-4001, 2014.

[10] B. K. namurthy, P. Gill, and M. Arlitt, "A few chirps about twitter," In: Proceedings of the $1^{\text {st }}$ Workshop on Online Social Networks, ACM, Washington, pp. 19-24, 2008

[11] M. E. J. Newman, and J. Park, "Why social networks are different from other types of networks," Physical Review E, vol. 3 pp. 036122:67-036122:77, 2003

[12] J. Weng, E. P. Lim, J. Jiang, and Q. He, "Twitterrank: finding topic-sensitive influential twitterers," In: Proceedings of the $3^{\text {rd }}$ ACM International Conference on Web Search and Data Mining, ACM, New York, 2010, pp. 221-231.

[13] B. Lars, and K. Jon, "Romantic partnerships and the dispersion of social ties: a network analysis of relationship status on facebook," In: Proceedings $17^{\text {th }}$ ACM Conference on Computer Supported Coopreative Work and Social Computing (CSCW), 2014, arXiv: 1310.6753 .

(C) Tao Shaohua; Licensee Bentham Open.

This is an open access article licensed under the terms of the (https://creativecommons.org/licenses/by/4.0/legalcode), which permits unrestricted, noncommercial use, distribution and reproduction in any medium, provided the work is properly cited. 